\title{
Characterisation of high rate plasticity in the uniaxial deformation of high purity copper at elevated temperatures
}

\author{
L.J. Lea ${ }^{\mathrm{a}, *}$, A.P. Jardine ${ }^{\mathrm{a}}$ \\ ${ }^{a}$ Cavendish Laboratory, JJ Thomson Ave, Cambridge, United Kingdom
}

\begin{abstract}
In uni-axial compression at strain rates above $10^{4} \mathrm{~s}^{-1}$, FCC metals exhibit a rapid increase in strength. Mechanisms proposed to be responsible for this transition can be broadly split into two categories; that mobile dislocation velocities become limited by quasi-viscous scattering from phonons, or that some change occurs in the evolution of the materials dislocation structure. The relative contribution of each mechanism is difficult to identify, in part due to a scarcity of experimental measurements in varying deformation conditions. In this paper, we perform uni-axial compression experiments that reach rates between $10^{4}$ and $10^{5} \mathrm{~s}^{-1}$, at temperatures between 300 and $600 \mathrm{~K}$. Analysis of the data at 0.1 strain shows both the absolute and relative levels of thermal softening increase with strain rate, an anomalous result in comparison to both existing models and measurements below the transition.
\end{abstract}

Keywords: Dislocations; High strain rate; Temperature; Phonon drag; Mechanical threshold

\section{Introduction}

Above uni-axial true strain rates, $\dot{\varepsilon}$, of $10^{4} \mathrm{~s}^{-1}$, FCC metals exhibit a rapid rise in strength; a phenomenon initially observed by Follansbee, Regazzoni and Kocks (Follansbee et al., 1984). The original measurements from their study are depicted in Figure 1, which shows how the stress required to continue deforming an oxygen free high conductivity (OFHC) copper varies at a fixed uni-axial strain, $\varepsilon$, as a function of strain rate. Importantly, in these measurements the strain rate remains approximately constant throughout deformation. The measurements have since been reproduced by numerous authors, and collated by Jordan et al. (2013).

The physical phenomena that have been proposed to explain the transition in behaviour can be broadly split into two categories. Firstly the speed of dislocations propagating strain is proposed to become limited by a quasi-viscous scattering from phonons in the lattice (Anderson et al., 2017; Nemat-Nasser and Li, 1998; Rusinek et al., 2010). The second is that a change occurs in the evolution of the internal dislocation structure (Armstrong and Li, 2015; Armstrong and Walley, 2008). Typically the change in dislocation evolution is predicted to result in an increase of the mechanical threshold (Follansbee and Kocks, 1988; Gao and Zhang, 2012), a state variable like parameter describing the stress required to initiate deformation in the absence of thermal effects (Follansbee, 2014).

A large number of both physical (Goto et al., 2000; Hansen et al., 2013; Hosseini and Kazeminezhad, 2009; Huang et al., 2009; Hunter and Preston, 2015; Langer et al., 2010; Preston et al., 2003) and phenomenological (Baig et al., 2013; Gao and Zhang, 2012; Gould and Goldthorpe, 2000; Khan and Liu, 2012; Molinari and Ravichandran, 2005; Regazzoni et al., 1987; Sung et al., 2010; Zerilli and Armstrong, 1987) models now exist, many more are collated by Salvado et al. (2017). In order

\footnotetext{
${ }^{*}$ Corresponding author

Email addresses: lewis.lea@cantab.net (L.J. Lea), apj24@cam.ac.uk (A.P. Jardine)
} 


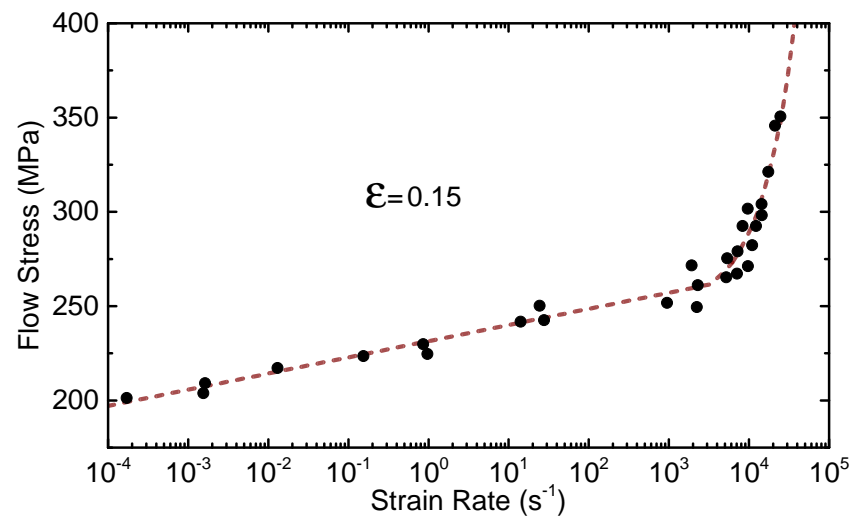

Figure 1: The flow strength of an $\mathrm{OFHC} \mathrm{Cu}$ at true 0.15 strain measured as a function of uniaxial strain rate, $\dot{\varepsilon}$. The dashed line is to guide the eye. Data taken from Follansbee et al. (Follansbee et al., 1984).

to discern between the plethora of available models, with varying emphasis on the two competing mechanisms, experimental measurements under many different conditions are required.

One approach for comparing models is through their predictions of material behaviour at varying temperatures and strain rates. These are useful as drag mechanisms have different temperature dependencies to structural ones. Despite the widespread use of the uniaxial stress geometry for model parametrisation, exemplified in data collation by Liang and Khan (1999), Jordan et al. (2013) and Hansen et al. (2013) measurements at elevated temperatures and rates above $10^{4} \mathrm{~s}^{-1}$ are sparse. The majority of measurements in that temperature and rate regime exist under conditions of shear strain (Frutschy and Clifton, 1998; Grunschel and Clifton, 2007) or the uni-axial strain conditions present in shock wave propagation (Chen et al., 2017; Gurrutxaga-Lerma et al., 2017; Kanel, 2014). Furthermore, in both cases measurements typically begin an order of magnitude above the transition rate. The aim of the current work is to extend miniaturised Hopkinson pressure bar experiments, used at very high rates, to elevated temperatures, allowing measurement of the response of metals in uni-axial stress conditions above $10^{4} \mathrm{~s}^{-1}$. Such measurements should act to connect thermal behaviour both below and well above the transition, and will provide better tests of how models address the transition in a uniaxial loading geometry.

In section 2 we will first outline the most basic picture describing the combination of the two mechanisms, before progressing on to a brief discussion of more sophisticated models, at each point discussing relevant experimental studies. Section 3 outlines the experimental arrangement, with emphasis on reducing errors at increased temperatures and on the limitations of the resulting measurements. Finally, in section 4 we will discuss the results obtained in terms of both viscous and thermal activation mechanisms, using rudimentary manipulations to avoid restricting observations to the framework of any one particular plasticity model.

\section{Background}

The general motion of a dislocation is typically modelled as being either stationary (pinned) or in transit. Acceleration time-scales are considered negligible, being of the order of 10 ps (Gorman et al., 1969). The drift velocity of a single dislocation is therefore described as

$\bar{v}=\frac{\lambda}{t_{\text {pin }}+t_{\text {tra }}}$,

where $\lambda$ is the "mean free path" between any two points at which a dislocation is pinned (Brown, 2012; Devincre et al., 2008), and varies with dislocation structure. The $t$ terms are the time-scales of pinning and transit respectively. 
Pinning, in its most simple form, is considered as when the strain fields of dislocations interact with those of immobile "forest" dislocations, typically by annihilating at a point and preventing motion until the lost dislocation segment can be regenerated (Hunter and Preston, 2015). Regeneration of the dislocation segment is typically modelled using transition state theory, commonly in an Arrhenius (Arrhenius, 1889) form,

$t_{\text {pin }} \approx \frac{1}{f_{D}} \exp \left[\frac{U\left(\hat{\sigma}, \sigma_{p}\right)}{k_{B} T}\right]$,

where $f_{D}$ is the vibrational frequency of the dislocation. $U$ is the remaining potential barrier given a structural "plastic" (Gould and Goldthorpe, 2000) or "threshold" (Gao and Zhang, 2012; Regazzoni et al., 1987) strength, $\sigma_{p}$, that would be required to deform the metal at $0 \mathrm{~K}$ and the applied stress $\hat{\sigma}$, typically after the subtraction of a small constant known as the "athermal" stress, $\sigma_{0}$, arising from "long range barriers" such as impurities and grain boundaries (Gao and Zhang, 2012). $T$ is the absolute temperature and $k_{B}$ is Boltzmann's constant. Due to false perturbation (Hunter and Preston, 2015) and relaxation (Hunter and Preston, 2015; Selyutina et al., 2016) considerations involved in this formulation, the model is incomplete. However it remains qualitatively similar enough to more rigorous approaches to allow use in the current discussion.

The true nature of pinning, especially in FCC metals, is much more complex. Different slip systems (Dequiedt et al., 2015) and dislocation reaction mechanisms (Kubin et al., 2003), such as locks (Hansen et al., 2013), pile-ups (Armstrong and Walley, 2008; Brown, 2012) and recovery Madec and Kubin (2017), must be accounted for. Whilst the mechanical threshold was originally posed as a single, constitutive quasi-state variable parameter (Follansbee and Kocks, 1988), contemporary approaches (Follansbee, 2014) attempt to individually model the many underlying processes. Models proposed by Austin and McDowell (2011) and Hansen et al. (2013) track the populations of immobile dislocations separated both by slip system and the mechanism in which they have been immobilised. Consequently, the relative numbers of mobile dislocations trapped in each type of barrier can be calculated. Such pictures inevitably have more complex temperature behaviour, and typically require a large number of parameters. Increasingly, computational simulations such as molecular dynamics (MD) (Olmsted et al., 2005) or discrete dislocation dynamics (DDD) (Madec and Kubin, 2017) are used to study individual mechanisms and subsequently allow for model parametrisation. Computational approaches come with their own difficulties, such as the current restrictions on simulation size hindering the complete portrayal of meso-scale patterning (Ispánovity et al., 2014), self-organisation (Brown, 2012) and grain-effects in polycrystals Richeton et al. (2005).

The transit term describes the time an unpinned dislocation takes to travel between two pinning sites against the viscous force. For dislocation speeds well below the speed of sound, the viscous force per unit length is proportional to the velocity of travel; $\hat{\sigma} b=M B(T) v$, where $b$ is the Burgers vector and $B(T)$ is the effective drag constant per unit dislocation length (Anderson et al., 2017). $M$ is the Taylor factor (Taylor, 1938), which relates the macroscopic uni-axial stress, $\sigma$, and strain, $\varepsilon$, to the local shear quantities, $\tau$ and $\gamma$, in a bulk polycrystal; $\sigma=M \tau$ and $\varepsilon=\gamma / M$. The Taylor factor has been measured as 3.06 for high purity copper (Rollett et al., 2004).

At ambient or raised temperatures the drag force is dominated by phonons scattering off the strain field both in the core and in the far field of the dislocation, which breaks down the harmonic nature of the lattice. Scattered phonons impart momentum to the dislocation, with no directional bias in the case of a stationary dislocation. However, for a moving dislocation there is a scattering bias against the direction of motion, which is approximately proportional to the dislocation velocity, providing it remains well below the speed of sound, $c_{s}$. Close to the sound speed, relativistic effects enhance the dislocation drag (Anderson et al., 2017; Armstrong et al., 2009)

$B(T) \rightarrow \frac{B(T)}{1-\left(v / c_{s}\right)^{2}}$

leading to a quadratic solution for velocity (Austin and McDowell, 2011) that asymptotically approaches the sound speed. The current work corresponds to sub relativistic velocities, where the resultant travel time is

$t_{\text {tra }} \approx \frac{M \lambda}{\hat{\sigma} b} B(T)$. 
The drift velocity can be combined with Orowan's law (Orowan, 1940) to calculate the achieved strain rate. Using a Taylor factor construction for present discussion, we arrive at the relation $\dot{\varepsilon}=\rho_{m} b \bar{v} / M$, where $\rho_{m}$ is the planar mobile dislocation density. In more explicit models, such as those of Austin and McDowell (2011) and Hansen et al. (2013), the shear strain rate of any system, $\mathrm{s}$, is $\dot{\gamma}_{s}=\rho_{m_{s}} b \bar{v}_{s}$ and the macroscopic strain is then computed by combining each of the individual systems (Hansen et al., 2013).

If drag is negligible (i.e. at low strain rates), for a given phenomenological model of the pinning barrier, the strain rate relation can be inverted (Follansbee and Kocks, 1988; Gao and Zhang, 2012; Hunter and Preston, 2015; Zerilli and Armstrong, 1987) to a material strength model, typically resulting in the form

$\sigma_{f}\left(\dot{\varepsilon}, T, \sigma_{p}\right)=\sigma_{0}+\phi(\dot{\varepsilon}, T) \sigma_{p}=\sigma_{0}+\hat{\sigma}$.

$\phi(\dot{\varepsilon}, T)$ is a scaling function varying between 0 and 1 describing the material strength, relative to the mechanical threshold, under the current conditions. The general behaviour of $\phi$ is as one would expect from typical metal behaviour, decreasing with temperature and increasing in a quasi-logarithmic manner with rate (Follansbee et al., 1984; Gould and Goldthorpe, 2000; Regazzoni et al., 1987). $\sigma_{p}$ describes the strength due to the deformation history of the metal (work hardening), acting similarly to a state variable (Gao and Zhang, 2012; Kocks and Mecking, 2003; Regazzoni et al., 1987). The majority of the strength in high purity FCC metals arises from path dependent work hardening effects, and accurately portraying path dependence is key to predicting their behaviour Follansbee (2014); Gould and Goldthorpe (2000).

As the rate increases, drag becomes increasingly important. If the applied stress exceeds the mechanical threshold Gurrutxaga-Lerma et al. (2017), or for high rates and temperatures close to melt Grunschel et al. (2012); Gurrutxaga-Lerma et al. (2017); Kanel (2014), pinning barriers are no longer capable of trapping dislocations and the system adopts a pure drag regime. For velocities well below the sound speed, the flow stress then takes the form

$\hat{\sigma}=\frac{M^{2}}{\rho_{m} b^{2}} B(T) \dot{\varepsilon}$.

In the viscous limit, the strength of the material becomes proportional to the viscosity coefficient, $B(T)$. In the relativistic limit, Austin and McDowell (2011) have shown that the applied stress and viscous coefficient only appear grouped in the form $\sigma / B(T)$, implying the proportionality holds.

Most prominent viscosity models suggest that above the Debye temperature drag effects are driven by anharmonic radiation from the dislocation core Lothe (1962) and the anharmonic scattering of lattice phonons from both the core and strain field of the dislocation Anderson et al. (2017). The viscosity coefficient is therefore proposed to inherit the temperature dependence of these processes, increasing with the thermal energy density in the lattice Anderson et al. (2017).

Different temperature sensitivities arise from different scattering mechanisms and regions of the phonon spectrum (Anderson et al., 2017). The majority of the viscous force occurs from anharmonic scattering processes known as "fluttering" (Al'shitz and Indenbom, 1975) and "phonon wind" (Brailsford, 1972), which are proportional to the absolute temperature. A small but significant temperature insensitive term arises from contributions due to phonons in the non linear region of the dispersion spectrum, known as "slow phonons" (Eshelby, 1949). Thermo-elastic effects (Weertman, 1996) lead to a quadratic temperature dependence, however in the temperature range of interest they are small even compared to slow phonon effects and are thus neglected. We can therefore describe the viscosity coefficient as $B(T) \approx B_{0}+B_{1} T\left(\approx B_{1} T\right)$. More detailed expressions for each term have been collated by Roos et al. (1999), which combined with standard material parameters estimate that between 300-600 $\mathrm{K}$, slow phonons $\left(B_{0}\right)$ are responsible for of order $10 \%$ of the overall drag. Such relations have been observed in simulations performed by Bitzek and Gumbsch $(2004,2005)$ and more recently Kuksin and Yanilkin (2013).

We find a significant difference in the temperature responses of the thermally activated and phonon drag regimes. Whilst the pinning regime thermally softens, in a purely viscous regime the specimen strength is predicted to increase approximately in proportion to the absolute temperature (Grunschel et al., 2012; Gurrutxaga-Lerma et al., 2017; Kanel, 2014; Kanel et al., 2001; Kuksin and Yanilkin, 2013; Zaretsky and Kanel, 2015). The transition is shown in Figure 2, that shows a sketch of the flow stress as a function of rate for two different temperatures as specimens enter a phonon limited regime. Such an inversion 
has been observed both in Hugoniot elastic limit measurements (Chen et al., 2017; Gurrutxaga-Lerma et al., 2017; Kanel, 2014; Zaretsky and Kanel, 2013) across a wide range of temperatures, and in shear-pressure experiments (Grunschel et al., 2012) close to melt. These experiments have provided some confidence in phonon drag mechanisms being active at high rates, with some quantitative discrepancies, proposed by Grunschel et al. (2012) to be due to interactions between moving dislocations. Many constitutive models which use drag do not address the temperature dependence of the viscosity coefficient (Salvado et al., 2017), largely due to the lack of parametrisation data.

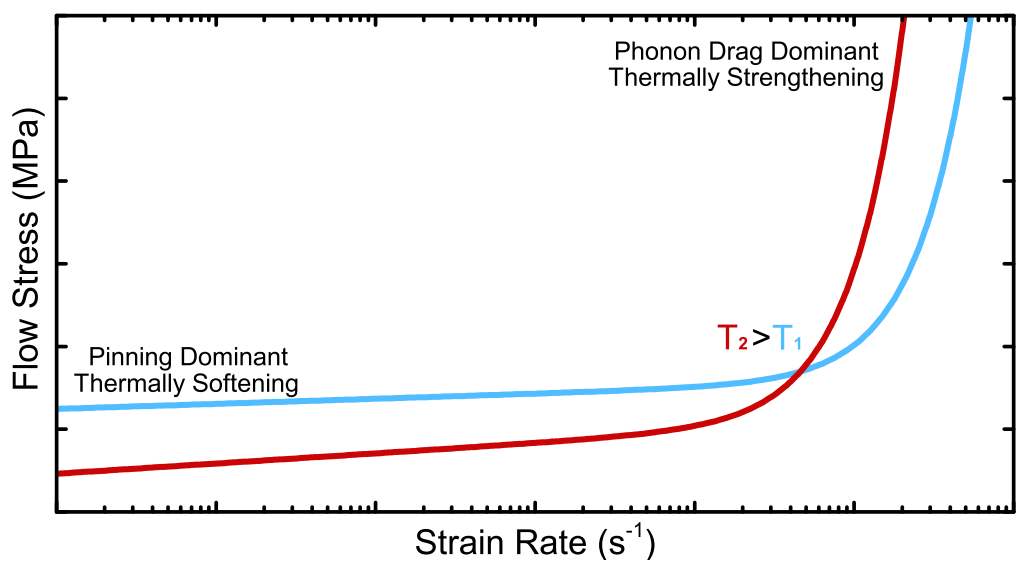

Figure 2: A sketch of the predicted flow stress of a pure FCC metal as a function of strain rate at two temperatures, $T_{2}>T_{1}$. Based on Figure 4 in Kanel et al. (2007) and Figure 1 (b) in Kuksin and Yanilkin (2013).

Despite the compiled experimental and computational evidence, the current understanding of the temperature dependence of drag effects is not complete. Outstanding phenomena exist such as those observed by Olmsted et al. (2005). They show using atomistic simulations that whilst edge dislocation velocities can be described purely as a function of $\sigma / B(T)$, screw dislocations only obey this relation up to approximately half of the shear wave speed, above which point the velocity depends solely on $\sigma$. The authors attribute this to radiative dissipation. However, the effect resides in the relativistic regime, at half of the sound speed, and should not be of concern in current study, just above the transition point.

At intermediate rates, such as those in this work, the scenario is more complex. Drag effects must be combined with the not yet negligible pinning barriers, and potentially even their increase with rate (Armstrong et al., 2009; Follansbee and Kocks, 1988). Models which consider the entirety of the observed increase above $10^{4} \mathrm{~s}^{-1}$ to arise from drag have proved unable to accurately capture observed increases in ductility (Armstrong and Li, 2015; Khan and Liu, 2012; Qiu, 2013) that accompany the transition. It would therefore appear that either a picture is required in which increases in mechanical threshold occur in parallel with drag effects, or one where they are superseded at high stresses, high temperatures or low dislocation densities. Another potential scenario proposed by Huang et al. (2009) is that phonon drag gives rise to an increased mechanical threshold, predicting early rates of work hardening to increase with temperature at sufficiently high rates. Given currently available experimental data and the variety of modelling approaches used, the magnitude of each contribution is difficult to verify.

Early experiments in the intermediate regime were performed by Kumar (1969) in the uniaxial stress geometry at rates up to $\dot{\varepsilon} \approx 2 \times 10^{3} \mathrm{~s}^{-1}$. Nemat-Nasser and Li (1998) extended measurements to $8000 \mathrm{~s}^{-1}$ for temperatures between 77 and $1100 \mathrm{~K}$ on OFHC Copper, remaining below the $10^{4} \mathrm{~s}^{-1}$ ambient temperature transition rate. The next available measurements in strain rate space are pressure-shear (Frutschy et al., 1998; Grunschel et al., 2012), which require models to simultaneously account for changes in geometry and potential new phenomena. Recently, similar rates have been achieved in extremely small SHPB apparatus, showing substantially lower flow stresses than in pressure-shear at ambient temperatures (Casem et al., 2018). 
Loading geometry effects can be partially mitigated using work such as that of Khan and Liu (2012), who have provided studies across many dynamic loading geometries and varying temperatures below the transition.

The aim of the current study is to provide currently unavailable elevated temperature measurements above $10^{4} \mathrm{~s}^{-1}$ in the uniaxial stress geometry, adding to the currently sparse non-shock measurements available across the strength transition. The measurements will provide a stronger basis with which to calibrate and verify increasingly rigorous physical models in a geometry compatable with the bulk of historical data (Hansen et al., 2013; Jordan et al., 2013; Nemat-Nasser and Li, 1998).

\section{Experimental Method}

Measurements were performed using a miniaturised split Hopkinson pressure bar (SHPB) apparatus (Gorham et al., 1992; Hopkinson, 1914; Kolsky, 1949; Kuhn and Medlin, 2000) shown in Figure 3. The specimen is sandwiched between two metal cylinders which are struck at one end by a projectile or "striker" bar. A compressive wave travels through the "input" bar and loads the specimen. As the specimen is (by design) smaller and softer than the bar material, it cannot transmit the full load and some amplitude is reflected along the input bar as a tensile wave. Meanwhile another wave, also defined by the size and strength of the specimen, is transmitted out through the "output" bar and is eventually contained in a momentum trap which moves away protecting the experiment. If the apparatus remains elastic throughout the experiment, the three waves can be analysed in different combinations to give complete force and displacement histories for both of the specimen faces.

The miniaturised SHPB approach was adopted as it presents one of few high rate methods of creating uniform strain, strain rate and temperature across a specimen, and uniform deformation rate throughout an experiment. Uniformity only occurs after an equilibration time, driven by a process known as "ring up", caused by reverberation in the sample driven by impedance mismatches at the bar-specimen interfaces. Ductile metals typically reach force equilibrium after three wave passes (Kuhn and Medlin, 2000), given their high sound speeds all but the earliest (typically $<5 \%$ strain) flow stress measurements are reliable. However, measurements such as elastic moduli are typically not. A second concern at higher rates is the rising edge of the input wave, which becomes shallow if significant levels of dispersion or misalignment are present (Gorham et al., 1992). To mitigate dispersive effects, the bars used had small diameters $(6.35 \mathrm{~mm})$ and were short $(25 \mathrm{~mm}$ length), sample free testing using a probe close to the input bar end found the rising edge rapid enough to perform measurements at 0.1 true strain.

Immediately before each test, specimens were induction heated to their target temperatures over a period of one minute, slowly enough to allow temperature measurements to be performed using a thermocouple on the corner of the output bar. To ensure the induction heater provided no electrical interference the specimens were heated to $10 \mathrm{~K}$ above the target temperature and let to air cool to the correct temperature. Between each change in testing temperature dummy specimens were placed in the system with physically attached thermocouples to both ensure the accuracy of the bar mounted thermocouple and to set the induction heater. The typical error in temperature due to this heating procedure was estimated as $5 \mathrm{~K}$.

The temperature of each specimen rises during an experiment, due to the work done and adiabatic nature of the deformation. The temperature change can be calculated from the work done per unit volume, which is equal to the integral of the stress strain curve (Gorham et al., 1992). Given the typical stress ranges expected, the temperature change is expected to be of the order of $100 \mathrm{~K}$ per unit strain. Full analysis requires iterative calculation to account for temperature variation during deformation. For our study we will mitigate the effect by restricting observations to low strains.

During each experiment, the surface velocities of the bars were probed using photon Doppler velocimetry (PDV) (Avinadav et al., 2011; Casem and Zellner, 2013; Lea and Jardine, 2016). Probe positions were at the midpoint of the input bar to allow separation of the incident and reflected waves, and $7 \mathrm{~cm}$ from the specimen on the output bar, the smallest distance satisfying Saint-Venant's principle (Ghiba, 2014) to avoid end effects. PDV probes have two significant benefits. Firstly, laser probes are not susceptible to damage, which typically hinders contact based strain gauge measurements at high impact speeds. Secondly, PDV measurements are unaffected by changes in the temperature of the bars; typical semiconductor or foil strain gauges both have temperature dependent responses. The bar material used was 718 Inconel, which has been shown by Walley (Walley, 2000) to have elastic properties negligibly dependent on temperature in the regime of interest. 


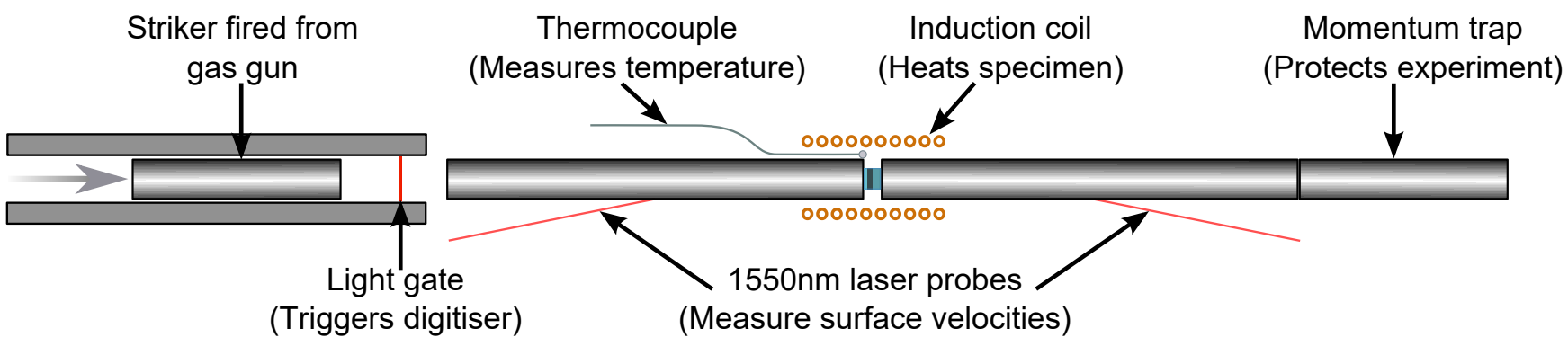

Figure 3: A schematic of the SHPB system used, outlined in detail in the body text. The bar system used was comprised of 6.35 mm diameter 718 Inconel bars. The input and output bars were both approximately $25 \mathrm{~cm}$ long whilst the striker bar and momentum trap were approximately $10 \mathrm{~cm}$ long.

PDV measurements were performed by reflecting $1550 \mathrm{~nm}$ lasers off both measurement points at $10^{\circ}$ angles from the surface. The laser light is doppler shifted due to both being received by and transmitted from the moving surface. The shifted light is mixed in a circulator, generating a slower oscillating beat pattern of the form

$I(t)=I_{0}+I_{1} \cos \left[2 f_{0} \frac{V(t)}{C_{0}}\right]$,

where $V(t)$ is the measured surface velocity, $f_{0}$ is the frequency of the laser and $C_{0}$ is the speed of light. The pattern is converted to a voltage in high speed photodiodes and digitised. The beat frequency, and thus velocity, is then extracted by breaking the trace into overlapping windows and fast Fourier transforming the data (Lea and Jardine, 2016).

After time shifting the pressure waves to account for the travel time between generation and measurement, the specimens deformation and force histories can be calculated. The specimen length can be calculated simply by integrating the velocity difference between the input and output faces,

$L(t)=L_{0}+\int V_{O}(t)-V_{I}(t)-V_{R}(t) d t$

where $L_{0}$ is the initial specimen length, and the $V$ terms correspond to the surface velocities caused by output, input and reflected waves respectively. Velocity vectors pointing from the input bar to the output bar are defined as positive. The specimen length is then converted to a true strain measurement using the definition $\varepsilon=\ln \left[L(t) / L_{0}\right]$. In compression the strain generated is negative, all measurements presented in this study show the magnitude of said strain.

The force at either specimen face can be calculated using the elastic behaviour of the bars (Kuhn and Medlin, 2000);

$F(t)=A_{B} Z_{B} \begin{cases}V_{O}(t), & \text { output face } \\ V_{I}(t)-V_{R}(t), & \text { input face }\end{cases}$

where $A_{B}$ is the area of the bars and $Z_{B}$ is their specific acoustic impedance. Typically the output force is used for specimen measurement and the input face to verify specimen equilibrium. Force measurements are converted into true stress measurements by dividing by the cross sectional area of the specimen, which varies during the experiment. As volume is approximately conserved in plastic deformation (Kuhn and Medlin, 2000), the specimen area as a function of time is generated using the relation $A(t)=A_{0}\left[L_{0} / L(t)\right]$, leading to a general form for flow stress as a function of time,

$\sigma=V_{O}(t) Z_{B} \frac{A_{B}}{A_{0}} \frac{L(t)}{L_{0}}$

The specimens themselves were right cylinders wire eroded from a plate of C103 grade oxygen free copper, with nominal purity greater than $99.95 \%$ and with the main impurity being lead. The cylinders were $3 \mathrm{~mm}$ in diameter and $500 \mu m$ thick. 
Micrography and EBSD study showed the grains to be of size 16-20 $\mu \mathrm{m}$ and with no strong preference in lattice orientation, quasi-static testing of five specimens gave repeatable results. The high strain rates involved in these experiments will lead to a significant inertial stress being superposed on top of the specimen strength measured by the output bar. General terms for inertia in compression have been derived by Gorham (Gorham, 1989; Jordan et al., 2013), and in the highest strain rate experiments the effect is expected to be approximately $10 \mathrm{MPa}$, small compared to the expected specimen strengths.

Powdered graphite was used to lubricate the bar ends, minimising frictional effects from radial expansion as the specimen compresses. The lubricant was chosen due to its consistent behaviour between ambient temperature and 600K. For the cylindrical geometry present in these experiments, and for small strains, the effect of friction on the flow stress of a specimen is described by the relation (Gorham et al., 1992)

$\sigma_{\text {measured }}=\sigma_{f}\left[1+\mu \frac{3 R(t)}{2 L(t)}\right] \approx \sigma_{f}\left[1+\mu \frac{3 R_{0}}{2 L_{0}} \exp (1.5 \varepsilon)\right]$,

where $R(t)$ is the specimen radius, and $\mu$ is the coefficient of friction. The value of $\mu$ was calibrated by performing equivalent tests on a number of specimens of varying aspect ratio and determining the value for the corrected stress-strain curves collapsed. The inferred value was $\mu=0.07 \pm 0.005$. For the presented experiments, all specimens have the same aspect ratio. Frictional effects therefore reduce to a strain dependent pre-factor, which is the same in every experiment. At high strains, effects such as the rolling round of the specimen edges complicate the nature of friction, again reinforcing that measurements are best considered at low strains, typically between 0.1 and 0.3 .

\section{Results \& Discussion}

A preliminary set of experiments were performed in both split and direct impact systems to determine at what rate the specimen material underwent the strength transition, as this is likely to be affected by grain size and purity. Figure 4 shows the same measurement of flow stress at fixed strain against log strain rate seen in Figure 1, however at 0.1 true strain and for the OF copper used in this study. The transition appears to occur at approximately $10^{4} \mathrm{~s}^{-1}$.

Based on room temperature measurements three target rates were chosen. Firstly, close to the transition at $2 \times 10^{4} \mathrm{~s}^{-1}$, where both structural and viscous thermal effects would be expected to cause intricate thermal behaviour. The second rate selected was $4 \times 10^{4} \mathrm{~s}^{-1}$, twice the transition rate and approaching twice the stress of the transition. At this rate viscous forces should be approaching an equal contribution and changes in thermal behaviour should be apparent. Finally, the maximum feasible rate available, given the experimental apparatus and temperature range, is $8 \times 10^{4} \mathrm{~s}^{-1}$, well above twice the transition strength - viscous effects should be clear at this rate.

Figure 5 shows the flow stresses measured as a function of strain and starting temperature for the achieved rates: $2.2 \times$ $10^{4} \mathrm{~s}^{-1}, 4.4 \times 10^{4} \mathrm{~s}^{-1}$ and $7.9 \times 10^{4} \mathrm{~s}^{-1}$. Each condition was tested in triplicate, the solid line presented for each set of conditions corresponds to the average flow stress measured for that strain, whilst the shaded region corresponds to the standard deviation in flow stress. The periodic nature of the standard deviation reflects the uncertainty being largely due to dispersion in the output wave. Despite measuring at the minimum distance permitted for a valid surface measurement the effects remain significant. A typical triplicate of individual results is shown as an inset.

In none of the measured stress-strain curves, presented in Figure 5, does the temperature dependency of the flow strength reverse (or vanish). The curves monotonically decrease in strength with increasing starting temperature. In this aspect the results are qualitatively consistent with the pressure-shear experiments of Frutschy et al. (1998). None of the experiments appear to correspond to a purely viscous regime

Continued thermal softening above the transition implies that despite the increase in flow strength, structural barriers remain a major strengthening mechanism. However, cursory analysis of the data at 0.1 strain shows discrepancies between the measured strengths and the expected outcomes of thermally activated and parallel drag and activation theories. For comparison 


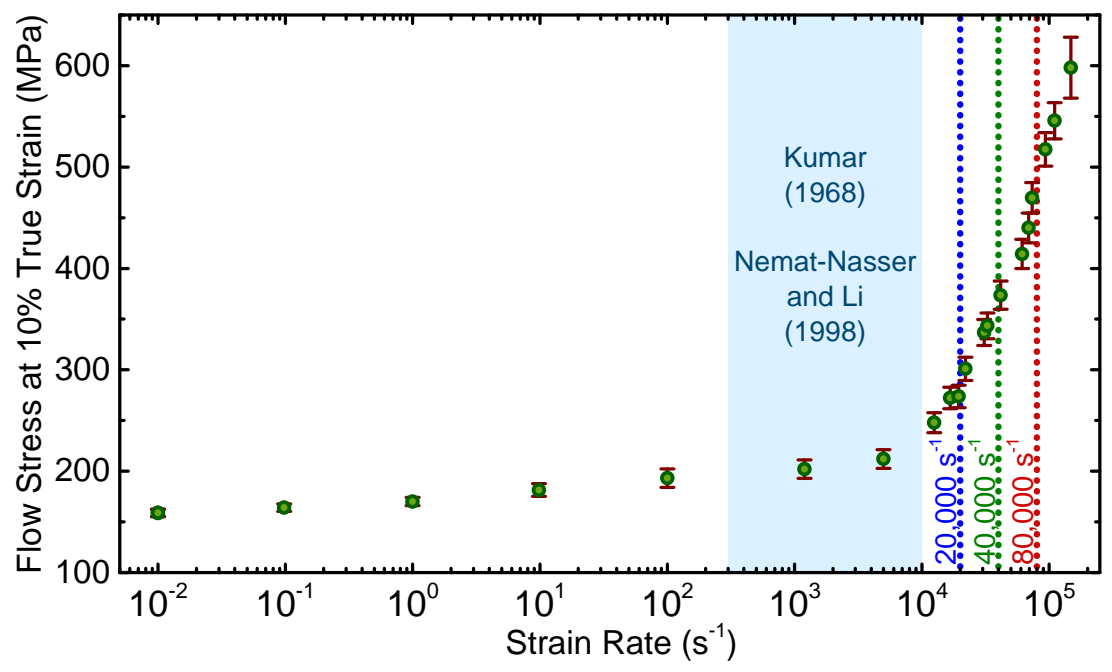

Figure 4: The flow stress at 0.1 true strain as a function of rate for the copper specimens in this study. The dotted lines signify the rates selected for study, discussed in the body text. The shaded region shows the range of rates previously studied by Kumar (1969) and Nemat-Nasser and Li (1998).

we sketch the form of $\phi$ proposed by Kocks et al. (1975), sketched in Figure 6, including their inferred exponents;

$\phi(\dot{\varepsilon}, T)=\frac{G(T)}{G(0)}\left[1-\frac{k_{B} T}{U_{0} G b^{3}} \ln \left(\frac{\dot{\varepsilon}_{0}}{\dot{\varepsilon}}\right)\right]^{3 / 2}$,

where $U_{0}$ is a dimensionless material parameter (Regazzoni et al., 1987), $\dot{\varepsilon}_{0}$ is some limiting strain rate taken to be $10^{7} \mathrm{~s}^{-1}$ (Regazzoni et al., 1987) and $G$ is the shear modulus. The relation is derived using a phenomenological form of $U\left(\hat{\sigma}, \sigma_{p}\right)$ which describes a quadratic remobilisation barrier with extended tails, accounting for the strain's far field (Kocks et al., 1975).

We note that as the rate increases, the various temperature curves converge, corresponding to a prediction of decreasing thermal softening with rate. Physically the behaviour can be rationalised from the fact that with increasing rates $\hat{\sigma}$ approaches $\sigma_{p}$, reducing the remaining barrier for thermal activation, and in turn the temperature sensitivity. Eventually the barrier reaches zero when the applied stress reaches the mechanical threshold. This behaviour is not directly observable in the stressstrain curves. Without any processing of the data it would appear that the specimens tested at $7.9 \times 10^{4} \mathrm{~s}^{-1}$ undergo greater absolute thermal softening than those at $2 \times 10^{4} \mathrm{~s}^{-1}$. The effect is shown in Figure 6 (b) which plots the flow stress at 0.1 true strain for each rate as a function of initial temperature, the higher rate measurements show a steeper thermal softening gradients. In a model with fixed mechanical threshold, the observed increase in thermal softening with strain rate at low strain cannot be explained. Similarly, any drag mechanism which is either independent of or increases with temperature also leads to the expectation of a reduction in thermal softening, rather than the observed increase. For comparrison, lower strain rate measurements were collected for the same material using a standard SHPB system at $10^{3} \mathrm{~s}^{-1}$ and an Instron testing system at $1 \mathrm{~s}^{-1}$ and $10^{-2} \mathrm{~s}^{-1}$. The lower strain rate measurements are also shown in Figure $6(\mathrm{~b})$.

The prediction of decreasing thermal softening with rate arises in many $\phi$ like terms. The model of Zerilli and Armstrong (1987) uses the form

$\phi(\dot{\varepsilon}, T)=\frac{G(T)}{G(0)} \exp \left[T\left(-\beta_{0}+\beta_{1} \ln \dot{\varepsilon}\right)\right]=\frac{G(T)}{G(0)}\left(\frac{\dot{\varepsilon}}{\dot{\varepsilon_{0}}}\right)^{\beta_{1} T}$,

where $\beta_{0}$ and $\beta_{1}$ are constant model parameters and $\ln \dot{\varepsilon}_{0}=\beta_{0} / \beta_{1}$. Gould and Goldthorpe (2000) extracted parameters for copper which provide a value of $\dot{\varepsilon}_{0} \approx 10^{7} \mathrm{~s}^{-1}$, in line with the value from Regazzoni et al. (1987). Importantly this 

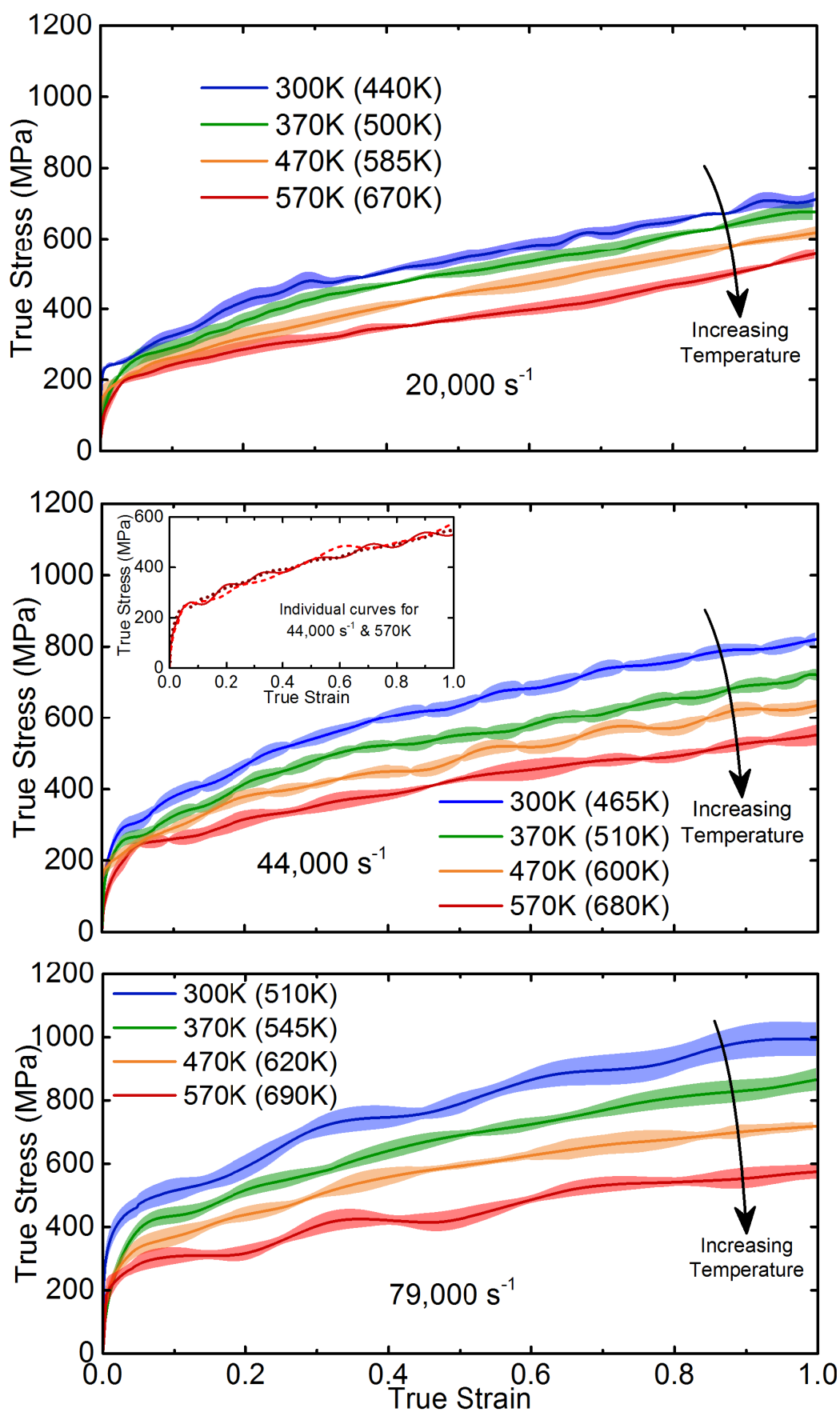

Figure 5: The flow stress of the OF copper described in the body text, as a function of strain for four temperatures and three strain rates. All of which are at or above the strength transition. Despite being well into the proposed viscous drag regime, the curves still monotonically decrease in strength with increasing temperature. 

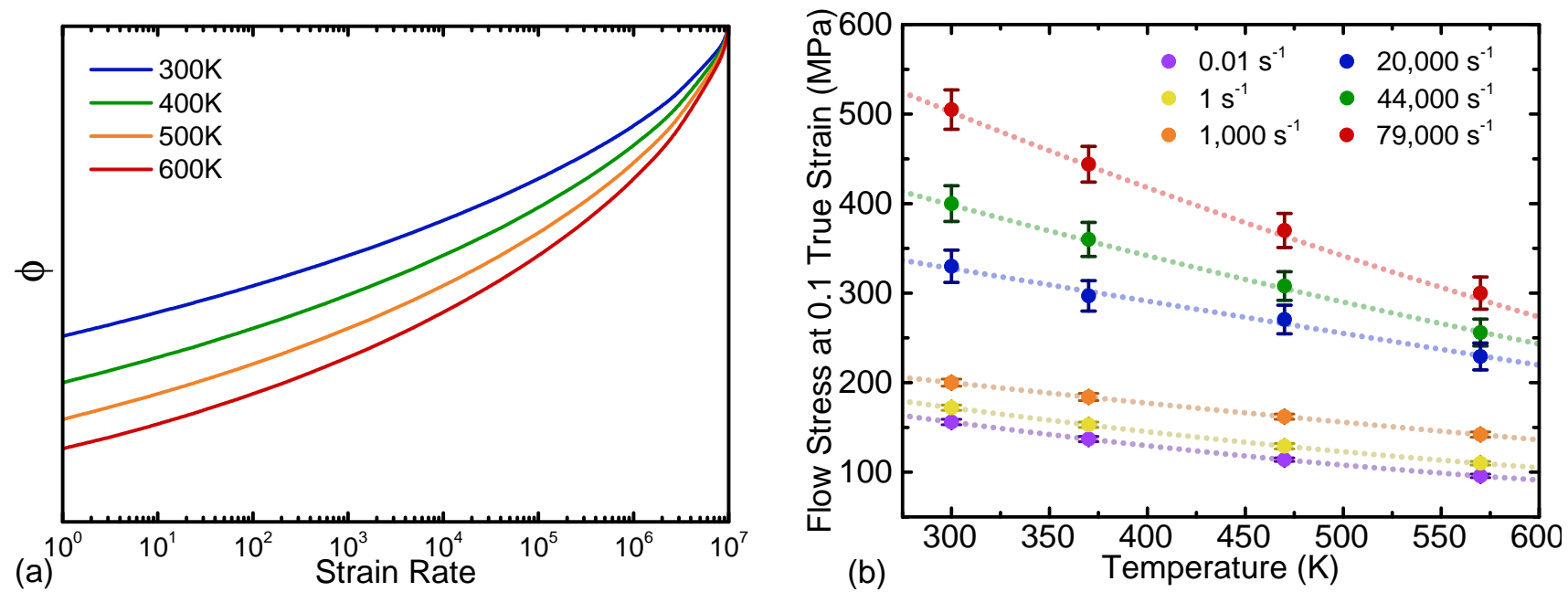

Figure 6: (a) A sketch of $\phi(\dot{\varepsilon}, T)$ as a function of rate for varying temperatures above ambient, based on the form from of Kocks et al. (1975). (b) The flow stress for each condition at 0.1 true strain as a function of temperature. The dotted lines on the graph are to guide the eye.

value is greater than the experimental range, meaning the pre-exponent is less than unity and the metal softens with increasing temperature. However, as the rate increases, the pre-exponent increases towards unity and thus the thermal softening decreases with rate. Again this is not consistent with observation, assuming no change in $\sigma_{p}$. The model of Hunter and Preston (2015) also predicts the same behaviour, evident most clearly in their publication in Figures 4 (a) below $10^{5} \mathrm{~s}^{-1}$ and 5 (d), where the curves approach even before collapsing on to their drag limited regions.

The increased thermal softening behaviour can partially be explained in models proposing increased work hardening, as increasing $\sigma_{p}$ may lead to greater absolute softening, even for reduced variations in $\phi$. If we assume we are observing some increase in work hardening due to strain rate alone, and that our measurements are at low enough strains that recovery effects (Brown and Bammann, 2012) for all specimens are the same, then $\sigma_{p}$ at fixed strain is purely a function of rate. We can therefore remove such effects by grouping the measurements by rate and then normalising each group to the corresponding ambient temperature measurement (an arbitrary choice), removing $\sigma_{p}$ as a common factor:

$\frac{\phi(\dot{\varepsilon}, T)}{\phi(\dot{\varepsilon}, 300 K)}=\frac{\hat{\sigma}(\dot{\varepsilon}, T)}{\hat{\sigma}(\dot{\varepsilon}, 300 K)}$.

We can then compare the normalised behaviour to typical model predictions of $\phi$.

Normalising $\phi$ to the $300 \mathrm{~K}$ measurement for each rate, we expect to see the curves shown in Figure 7 (a). In this scenario, the curves all begin at unity, however the lower rates decrease more quickly, due to their higher temperature sensitivity. Thus, if the experimentally observed strength increase is due to some general, purely rate dependent increase in work hardening, we expect the level of thermal softening, normalised to the ambient temperature flow stress at that rate, to decrease with increasing rate. Figures 7 (b) and (c) show the measurements taken at 0.1 true strain (presented earlier in Figure 6 (b)) normalised to each rates ambient temperature measurement. Whilst measurements below the transition order in the expected manner, (Figure 7 (b)), above the transition (Figure 7 (c)) the order is reversed. We see that even after normalisation, the $7.9 \times 10^{4} \mathrm{~s}^{-1}$ specimens still show the greatest levels of softening. The highest rates undergo increased thermal softening both in absolute and relative terms.

There are three potential sources for such a discrepancy. Firstly, current models for $\phi$ may be inaccurate above the transition. There may be a phenomenological change in how structural barriers are surpassed, either leading to an increasing temperature sensitivity in $\phi$ with rate, or rendering the $\phi$ based formulation unusable. 


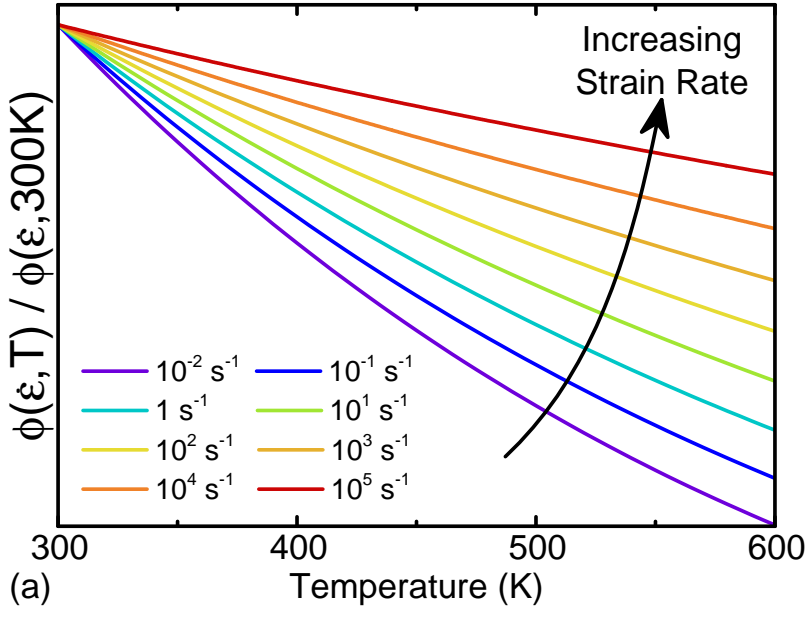

(a)

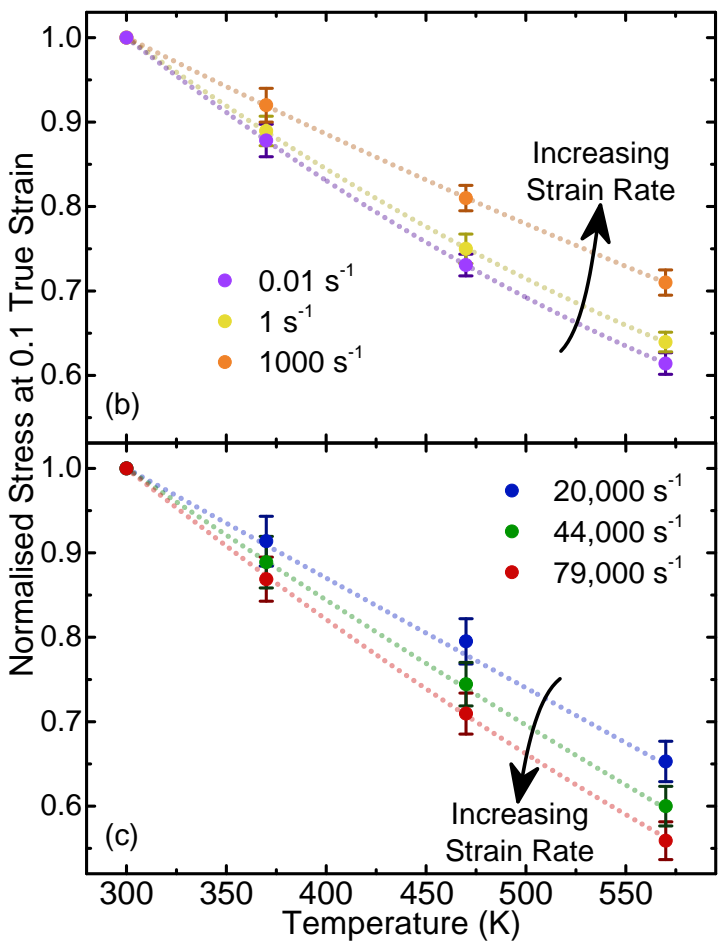

Figure 7: (a) A sketch predicted variation in $\phi$ with temperature normalised to the value of $\phi$ at room temperature for a variety of rates between $10^{4} \mathrm{~s}^{-1}$ and $10^{5} \mathrm{~s}^{-1}$, based on the form of Follansbee et al. (Regazzoni et al., 1987). (b) and (c) show the flow stress at 0.1 strain for each rate and temperature, as a fraction of the ambient temperature strength for that rate, after the removal of the athermal term $\left(\sigma_{0}\right)$.

Secondly, whilst specifics surrounding the process responsible for the transition are unknown, many strain rate dependent processes in metals exhibit "time-temperature superposition" effects. Typically, increasing the specimen temperature has effects similar to decreasing the applied strain rate. If the specimen strength increases due to a rate dependent increase in work hardening, increasing temperature may affect the underlying process in a way analogous to performing work hardening at a lower rate.

Thirdly, if the strength increase arises from an increased rate of work hardening, higher rate specimens may experience an earlier onset of "stage III" work hardening (Brown and Bammann, 2012; Brown, 2012), in which dynamic recovery (or in some models exhaustion) effects progressively reduce the rate of work hardening to a small fraction of that in stage II. The stress at which work hardening transfers to stage III (or in a more continuous picture of work hardening, the plateau stress) decreases with temperature. If higher rate specimens undergo greater initial work hardening, then at a fixed strain their higher mechanical threshold is likely to be more greatly affected by temperature dependent saturative effects. Saturative effects were assumed minor in the present analysis due to the measurements used being extracted relatively low strain, however, for increased rates of work hardening, the assumption may no longer hold. It should be noted that this scenario is already predicted by many state-variable based models (Follansbee and Kocks, 1988; Gao and Zhang, 2012; Goto et al., 2000; Hunter and Preston, 2015).

We now address the entire set of stress strain measurements in general. As the specimen deforms and approaches the plateau stress, adiabatic heating of the specimen, outlined in section 3 , will play a significant role. As the temperature increases both the $\phi$ terms would, based on current observation, be expected to decrease, as will the plateau stress to which the curves 
progress. Importantly, as the work done in plastic deformation is different for each experiment, so will the temperature rise as a function of strain. For general strains, more complex analysis is required to track the temperature changes and the increasing effects of recovery. The more complex analysis typically requires a constitutive model as a framework and thus will be left to such studies. In Figure 5, the final temperature at unit strain in each experiment is included in brackets next to the starting temperature, however the reader is reminded these temperature changes are much greater than those expected at the 0.1 strain discussed. A concern which cannot easily be accounted for in material models is the effect friction at large strains (above the 0.1 strain used for discussion), which is not necessarily consistent between experiments and difficult to quantitatively account for. As both the strain and flow stress become large, the potential for twinning may arise. Based on the observations of Meyers et al. (2001), at 0.8 strain and for $20 \mu \mathrm{m}$ grains, twinning at $10^{5} \mathrm{~s}^{-1}$ is expected at a temperature of approximately $25 \mathrm{~K}$, and becomes decreasingly likely with increasing grain size and temperature. We therefore assume twinning to be a minor contribution to the observed behaviour. Due to experimental equilibration, very low strain data (less than 0.05) cannot be discussed with confidence. Behaviour at very early stages of deformation, and thus potential drag determined behaviours expected at low dislocation densities, cannot be usefully studied.

In order to fully understand the observed increase in thermal softening, and indeed the strength transition itself, measurements are required which separate instantaneous $(\phi)$ and structural $\left(\sigma_{p}\right)$ effects to allow detailed studies of the individual mechanisms, rather than their combined effects. The results of the present study emphasise the importance of the threshold stress as a quasi-state variable in FCC metal deformation, and the need to properly understand its behaviour in the absence of dynamic effects.

\section{Conclusions}

A series of oxygen free copper specimens were tested, providing previously unavailable uniaxial stress data for elevated temperatures at rates around and immediately above the onset of the materials strength transition at $10^{4} \mathrm{~s}^{-1}$. Instead of a reduction in thermal softening with rate, predicted by phonon drag and many $\phi$ type terms, the level of both absolute and relative thermal softening increased with rate. Such observations would appear consistent with an increase in mechanical threshold, however rudimentary corrections for an increased in threshold do not completely account for the observed increases in thermal softening. Several potential explanations for the remaining discrepancy were outlined. We expect the extension of uniaxial stress measurements provided in this work will prove important in the parametrisation and verification of contemporary models. In order to properly understand the behaviour of the materials, further experiments are required to separate instantaneous $(\phi)$ and structural $\left(\sigma_{p}\right)$ effects.

\section{Acknowledgements}

This research was supported by grants from QinetiQ and the Engineering and Physical Sciences Research Council, as per EPSRC open data requirements, the data presented in this article can be found at https://doi.org/10.17863/CAM.12338.

The analysis of PDV data was performed using software written by N. Taylor of the Surface, Microstructure and Fracture group at the Cavendish Laboratory.

The authors would like to thank P.J. Gould and P.D. Church for their interest.

Al'shitz, V., Indenbom, V., 1975. Dynamic dragging of dislocations. Soviet Physics Uspekhi 18 (1), 1.

URL https://doi .org/10.1070/PU1975v018n01ABEH004689

Anderson, P., Hirth, J., Lothe, J., 2017. Theory of Dislocations, Third Edition. Cambridge University Press.

Armstrong, R., Arnold, W., Zerilli, F., 2009. Dislocation mechanics of copper and iron in high rate deformation tests. Journal of Applied Physics 105 (2), 023511.

URL http://scitation.aip.org/content/aip/journal/jap/105/2/10.1063/1.3067764 
Armstrong, R., Li, Q., 2015. Dislocation Mechanics of High-Rate Deformations. Metallurgical and Materials Transactions A (1).

URL http://link. springer . com/10.1007/s11661-015-2779-6

Armstrong, R., Walley, S., may 2008. High strain rate properties of metals and alloys. International Materials Reviews 53 (3), $105-128$.

URL http://www.ingentaconnect.com/content/maney/imr/2008/00000053/00000003/art00001http: //www.maneyonline.com/doi/abs/10.1179/174328008X277795

Arrhenius, S., 1889. Z. Phys. Chem.

Austin, R., McDowell, D., 2011. A dislocation-based constitutive model for viscoplastic deformation of fcc metals at very high strain rates. International Journal of Plasticity 27 (1), $1-24$.

URL http://www. sciencedirect.com/science/article/pii/S0749641910000380

Avinadav, C., Ashuach, Y., Kreif, R., 2011. Interferometry-based kolsky bar apparatus. Review of Scientific Instruments $82(7), 073908$.

URL http://dx.doi .org/10.1063/1.3615243

Baig, M., Khan, A. S., Choi, S.-H., Jeong, A., 2013. Shear and multiaxial responses of oxygen free high conductivity (ofhc) copper over wide range of strain-rates and temperatures and constitutive modeling. International Journal of Plasticity 40, $65-80$.

URL http://wWW. sciencedirect.com/science/article/pii/S074964191200112X

Bitzek, E., Gumbsch, P., 2004. Atomistic study of drag, surface and inertial effects on edge dislocations in face-centered cubic metals. Materials Science and Engineering: A 387, 11 - 15, 13th International Conference on the Strength of Materials. URL http://wWw. sciencedirect. com/science/article/pii/S0921509304004927

Bitzek, E., Gumbsch, P., 2005. Dynamic aspects of dislocation motion: atomistic simulations. Materials Science and Engineering: A 400, $40-44$, dislocations 2004.

URL http://www.sciencedirect. com/science/article/pii/S0921509305002571

Brailsford, A., 1972. Anharmonicity contributions to dislocation drag. Journal of Applied Physics 43 (4), 1380-1393.

URL http://dx.doi.org/10.1063/1.1661329

Brown, A., Bammann, D., 2012. Validation of a model for static and dynamic recrystallization in metals. International Journal of Plasticity $3233,17-35$.

URL http://www.sciencedirect.com/science/article/pii/S0749641911001963

Brown, L., nov 2012. Constant intermittent flow of dislocations: central problems in plasticity. Materials Science and Technology 28 (11), 1209-1232.

URL http://www. maneyonline.com/doi/abs/10.1179/174328412X13409726212768

Casem, D. T., Ligda, J. P., Schuster, B. E., Mims, S., 2018. High-Rate Mechanical Response of Aluminum Using Miniature Kolsky Bar Techniques. Springer International Publishing, Cham, pp. 147-153.

URL https://doi .org/10.1007/978-3-319-62956-8_25

Casem, D. T., Zellner, M. B., mar 2013. Kolsky Bar Wave Separation Using a Photon Doppler Velocimeter. Experimental Mechanics 53 (8), 1467-1473.

URL http://link. springer . com/10.1007/s11340-013-9735-4 
Chen, L., Swift, D., Austin, R., Florando, J., Hawreliak, J., Lazicki, A., Saculla, M. D., Eakins, D., Bernier, J. V., Kumar, M., 2017. Temperature dependence of dynamic deformation in fcc metals, aluminum and invar. AIP Conference Proceedings $1793(1), 110008$.

URL http://aip.scitation.org/doi/abs/10.1063/1.4971671

Dequiedt, J., Denoual, C., Madec, R., 2015. Heterogeneous deformation in ductile fcc single crystals in biaxial stretching: the influence of slip system interactions. Journal of the Mechanics and Physics of Solids 83, $301-318$.

URL http://wwW. sciencedirect.com/science/article/pii/S0022509615001313

Devincre, B., Hoc, T., Kubin, L., 2008. Dislocation mean free paths and strain hardening of crystals. Science 320 (5884), $1745-1748$.

URL http://science.sciencemag.org/content/320/5884/1745

Eshelby, J., 1949. Uniformly moving dislocations. Proceedings of the Physical Society. Section A 62 (5), 307.

URL https://doi .org/10.1088/0370-1298/62/5/307

Follansbee, P., 2014. On the definition of state variables for an internal state variable constitutive model describing metal deformation. Materials Sciences and Applications 5, 603-609.

URL http://dx.doi .org/10.4236/msa.2014.58062

Follansbee, P., Kocks, U., 1988. A constitutive description of the deformation of copper based on the use of the mechanical threshold stress as an internal state variable. Acta Metallurgica 36 (1), 81-93.

URL http: //linkinghub. elsevier .com/retrieve/pii/0001616088900302

Follansbee, P., Regazzoni, G., Kocks, U., 1984. The transition to drag-controlled deformation in copper at high strain rates. Mechanical Properties at High Rates of Strain 70, 71-80.

Frutschy, K., Clifton, R., 1998. High-temperature pressure-shear plate impact experiments on ofhc copper. Journal of the Mechanics and Physics of Solids 46 (10), 1723 - 1744.

URL http://wWw. sciencedirect.com/science/article/pii/S0022509698000556

Frutschy, K. J., Clifton, R. J., Mello, M., 1998. High-temperature pressure-shear plate impact studies on ofhc copper and pure wc. AIP Conference Proceedings 429 (1), 463-466.

URL http://aip.scitation.org/doi/abs/10 . 1063/1.55547

Gao, C., Zhang, L., 2012. Constitutive modelling of plasticity of fcc metals under extremely high strain rates. International Journal of Plasticity 3233, $121-133$.

URL http://www. sciencedirect.com/science/article/pii/S0749641911001914

Ghiba, I.-D., 2014. Saint-Venant's Principle. Springer Netherlands, Dordrecht, pp. 4255-4264.

URL http://dx.doi.org/10.1007/978-94-007-2739-7_258

Gorham, D., Pope, P., Field, J., jul 1992. An Improved Method for Compressive Stress-Strain Measurements at Very High Strain Rates. Proceedings of the Royal Society A: Mathematical, Physical and Engineering Sciences 438 (1902), 153-170. URL http://rspa.royalsocietypublishing.org/cgi/doi/10.1098/rspa.1992.0099

Gorham, D. A., 1989. J. Phys. D: Appl. Phys 22, 1888-1893.

Gorman, J. A., Wood, D. S., Jr., T. V., 1969. Mobility of dislocations in aluminum. Journal of Applied Physics 40 (2), $833-$ 841.

URL http://dx . doi .org/10.1063/1.1657472 
Goto, D. M., Garrett, R. K., Bingert, J. F., Chen, S. R., Gray, G. T., 2000. The mechanical threshold stress constitutive-strength model description of hy-100 steel. Metallurgical and Materials Transactions A 31 (8), 1985-1996.

URL http://dx.doi .org/10.1007/s11661-000-0226-8

Gould, P., Goldthorpe, B., 2000. A path-dependent constitutive model for gilding copper. J. Phys. IV 10.

URL https://doi .org/10.1051/jp4:2000907

Grunschel, S., Clifton, R., Dec 2007. Dynamic plastic response of aluminum at temperatures approaching melt. Metallurgical and Materials Transactions A 38 (12), 2885-2890.

URL https://doi.org/10.1007/s11661-007-9330-3

Grunschel, S., Clifton, R., Jiao, T., 2012. Shearing resistance of aluminum at high strain rates and at temperatures approaching melt. AIP Conference Proceedings 1426 (1), 1335-1338.

URL http://aip.scitation.org/doi/abs/10.1063/1.3686527

Gurrutxaga-Lerma, B., Shehadeh, M., Balint, D., Dini, D., Chen, L., Eakins, D., 2017. The effect of temperature on the elastic precursor decay in shock loaded fcc aluminium and bcc iron. International Journal of Plasticity 96, $135-155$. URL http://wWw. sciencedirect.com/science/article/pii/S074964191730150X

Hansen, B., Beyerlein, I., Bronkhorst, C., Cerreta, E., Dennis-Koller, D., 2013. A dislocation-based multi-rate single crystal plasticity model. International Journal of Plasticity 44 (Complete), 129-146.

Hopkinson, B., 1914. A method of measuring the pressure produced in the detonation of high explosives or by the impact of bullets. Philosophical Transactions of the Royal Society of London. Series A, Containing Papers of a Mathematical or Physical Character 213, 437-456.

URL http://www . jstor .org/stable/91070

Hosseini, E., Kazeminezhad, M., 2009. A hybrid model on severe plastic deformation of copper. Computational Materials Science 44 (4), $1107-1115$.

URL http://www. sciencedirect.com/science/article/pii/S0927025608003637

Huang, M., Riverz-Díaz-del Castillo, P., Bouaziz, O., 2009. A constitutive model for high strain rate deformation in fcc metals based on irreversible thermodynamics. Mech. Mater. 41, 982-988.

Hunter, A., Preston, D. L., 2015. Analytic model of the remobilization of pinned glide dislocations from quasi-static to high strain rates. International Journal of Plasticity 70, 1-29.

URL http://dx.doi.org/10.1016/j.ijplas.2015.01.008

Ispánovity, P., Laurson, L., Zaiser, M., Groma, I., Zapperi, S., Alava, M., jun 2014. Avalanches in 2D Dislocation Systems: Plastic Yielding Is Not Depinning. Physical Review Letters 112 (23), 235501.

URL http://link.aps.org/doi/10.1103/PhysRevLett.112.235501

Jordan, J., Siviour, C., Sunny, G., Bramlette, C., Spowart, J., 2013. Strain rate-dependant mechanical properties of ofhc copper. Journal of Materials Science 48 (20), 7134-7141.

URL http://dx.doi.org/10.1007/s10853-013-7529-9

Kanel, G., Fortov, V., Razorenov, S., 2007. Shock waves in condensed-state physics. Physics-Uspekhi 50 (8), 771. URL http://stacks.iop.org/1063-7869/50/i=8/a=R01

Kanel, G. I., 2014. Unusual behaviour of usual materials in shock waves. Journal of Physics: Conference Series 500 (1), 012001.

URL http: //stacks. iop.org/1742-6596/500/i=1/a=012001 
Kanel, G. I., Razorenov, S. V., Baumung, K., Singer, J., 2001. Dynamic yield and tensile strength of aluminum single crystals at temperatures up to the melting point. Journal of Applied Physics 90 (1), 136-143.

URL http://dx.doi.org/10.1063/1.1374478

Khan, A., Liu, H., 2012. Strain rate and temperature dependent fracture criteria for isotropic and anisotropic metals. International Journal of Plasticity $37,1-15$.

URL http://www. sciencedirect.com/science/article/pii/S0749641912000137

Kocks, U., Argon, A., Ashby, M., 1975. Thermodynamics and kinetics of slip. Prog. Mater. Sci. 19, 1-288.

Kocks, U., Mecking, H., jan 2003. Physics and phenomenology of strain hardening: The FCC case. Progress in Materials Science 48 (3), 171-273.

URL http://linkinghub.elsevier.com/retrieve/pii/S0079642502000038

Kolsky, H., 1949. An investigation of the mechanical properties of materials at very high rates of loading. Proceedings of the Physical Society. Section B 676.

URL http://iopscience.iop.org/0370-1301/62/11/302

Kubin, L., Madec, R., Devincre, B., 2003. Dislocation intersections and reactions in fcc and bcc crystals. MRS Proceedings 779 .

Kuhn, H., Medlin, D. (Eds.), 2000. ASM Handbook Vol 8: Mechanical Testing and Evaluation. Ch. 6.

Kuksin, a. Y., Yanilkin, a. V., may 2013. Atomistic simulation of the motion of dislocations in metals under phonon drag conditions. Physics of the Solid State 55 (5), 1010-1019. URL http://link. springer .com/10.1134/S1063783413050193

Kumar, A., 1969. Viscous Drag on Dislocations at High Strain Rates in Copper. Journal of Applied Physics 40 (9), 3475. URL http://scitation.aip.org/content/aip/journal/jap/40/9/10.1063/1.1658222

Langer, J., Bouchbinder, E., Lookman, T., 2010. Thermodynamic theory of dislocation-mediated plasticity. Acta Materialia 58 (10), $3718-3732$.

URL http://www.sciencedirect.com/science/article/pii/S1359645410001540

Lea, L., Jardine, A., 2016. Application of photon doppler velocimetry to direct impact hopkinson pressure bars. Review of Scientific Instruments 87 (2), 023101.

URL http://dx.doi.org/10.1063/1.4940935

Liang, R., Khan, A. S., 1999. A critical review of experimental results and constitutive models for bcc and fcc metals over a wide range of strain rates and temperatures. International Journal of Plasticity 15 (9), $963-980$.

URL http://www. sciencedirect. com/science/article/pii/S0749641999000212

Lothe, J., 1962. Theory of dislocation mobility in pure slip. Journal of Applied Physics 33 (6), 2116-2125.

URL http://dx. doi.org/10.1063/1.1728907

Madec, R., Kubin, L. P., 2017. Full length article. Acta Materialia 126 (Complete), 166-173.

Meyers, M., Vhringer, O., Lubarda, V., 2001. The onset of twinning in metals: a constitutive description. Acta Materialia 49 (19), $4025-4039$.

URL http://www.sciencedirect.com/science/article/pii/S1359645401003007 
Molinari, A., Ravichandran, G., 2005. Constitutive modeling of high-strain-rate deformation in metals based on the evolution of an effective microstructural length. Mechanics of Materials, $737-752$.

URL http://www. sciencedirect.com/science/article/pii/S0167663604001097

Nemat-Nasser, S., Li, Y., 1998. Flow stress of f.c.c. polycrystals with application to ofhc cu. Acta Materialia 46 (2), 565 577.

URL http://www.sciencedirect.com/science/article/pii/S1359645497002309

Olmsted, D., Hector, L., Curtin, W., Clifton, R., 2005. Atomistic simulations of dislocation mobility in al, ni and al/mg alloys. Modelling and Simulation in Materials Science and Engineering 13 (3), 371.

URL http: //stacks . iop . org/0965-0393/13/i=3/a=007

Orowan, E., 1940. Problems of plastic gliding. Proceedings of the Physical Society 52 (1), 8.

URL http: //stacks.iop.org/0959-5309/52/i=1/a=303

Preston, D., Tonks, D., Wallace, D., 2003. Model of plastic deformation for extreme loading conditions. Journal of Applied Physics, 211-220.

URL http://dx.doi .org/10.1063/1.1524706

Qiu, H., 2013. Integration of temperature, stress state, and strain rate for the ductility of ductile metals. Materials Transactions 54 (4), 626-629.

Regazzoni, G., Kocks, U., Follansbee, P., 1987. Dislocation kinetics at high strain rates. Acta Metallurgica 35 (12), 2865 2875.

URL http://www. sciencedirect.com/science/article/pii/0001616087902859

Richeton, T., Weiss, J., Louchet, F., 2005. Breakdown of avalanche critical behaviour in polycrystalline plasticity. Nature Materials, 465-469.

Rollett, A., Humphreys, F., Rohrer, G. S., Hatherly, M., 2004. Recrystallization and related annealing phenomena. Elsevier.

Roos, A., De Hosson, J., Cleveringa, H., Van der Giessen, E., 1999. Fast-moving dislocations in high strain rate deformation. University Library Groningen.

URL http://hdl.handle.net/11370/a0c6e779-88a6-4b96-909c-c01c42284332

Rusinek, A., Rodrguez-Martnez, J., Arias, A., 2010. A thermo-viscoplastic constitutive model for $\{\mathrm{FCC}\}$ metals with application to $\{$ OFHC $\}$ copper. International Journal of Mechanical Sciences 52 (2), 120 - 135, \{SPECIAL\} ISSUE: Advances in Modeling and Evaluation of Materials in Honor of Professor Tomita.

URL http://www. sciencedirect.com/science/article/pii/S0020740309001350

Salvado, F., Teixeira-Dias, F., Walley, S., Lea, L., Cardoso, J., 2017. A review on the strain rate dependency of the dynamic viscoplastic response of fcc metals. Progress in Materials Science 88, 186- 231.

URL http://www. sciencedirect.com/science/article/pii/S0079642517300397

Selyutina, N., Borodin, E., Petrov, Y., Mayer, A., 2016. The definition of characteristic times of plastic relaxation by dislocation slip and grain boundary sliding in copper and nickel. International Journal of Plasticity 82, $97-111$.

URL http://www. sciencedirect.com/science/article/pii/S0749641916300225

Sung, J. H., Kim, J. H., Wagoner, R., 2010. A plastic constitutive equation incorporating strain, strain-rate, and temperature. International Journal of Plasticity 26 (12), $1746-1771$.

URL http://www. sciencedirect. com/science/article/pii/S0749641910000276 
Taylor, G., 1938. Plastic strain in metals. J. Inst. Metals 62, 307-324.

Walley, S., 2000. Hopkinson bar testing at non-ambient temperatures. DYMAT 2000 Booklet.

URL www . dymat .org

Weertman, J., 1996. Dislocation based fracture mechanics. World Scientific.

Zaretsky, E. B., Kanel, G. I., 2013. Response of copper to shock-wave loading at temperatures up to the melting point. Journal of Applied Physics 114 (8).

URL http://scitation. aip.org/content/aip/journal/jap/114/8/10.1063/1.4819328

Zaretsky, E. B., Kanel, G. I., 2015. Yield stress, polymorphic transformation, and spall fracture of shock-loaded iron in various structural states and at various temperatures. Journal of Applied Physics 117 (19), 195901.

URL http://dx.doi .org/10.1063/1.4921356

Zerilli, F., Armstrong, R., 1987. Dislocation-mechanics-based constitutive relations for material dynamics calculations. Journal of Applied Physics 61 (5), 1816-1825.

URL http://dx.doi .org/10.1063/1.338024 